\title{
A simple and robust model to explain convex corner undercutting in wet bulk micromachining
}

\author{
Prem Pal and Sajal Sagar Singh
}

\begin{abstract}
In this paper, a simple and robust model is presented to explain the main reason behind undercutting at convex corners and no-undercutting at concave corners. The etch rate of the tangent plane at convex corner and the role of dangling bond in etching process are utilized to explain the undercutting at convex corner and the no-undercutting at concave corner, respectively. The present model shows that $\{110\}$ is the tangent plane at convex corner which exhibits higher etch rate than the neighboring $\{111\}$ plane in all types of anisotropic etchants; consequently the undercutting occurs at convex corners. The absence of dangling bonds at concave corner prevents the undercutting there. Moreover, the same model explains the reason of very less undercutting when the etching is carried out in surfactant-added tetramethylammonium hydroxide (TMAH).
\end{abstract}

Keywords: Convex and concave corners; MEMS; Silicon; Wet anisotropic etching; $\mathrm{KOH}$; TMAH

\section{Introduction}

The wafer manufacturing industries commonly produce the silicon wafers with three principle orientations namely $\{111\},\{110\}$ and $\{100\}$. Out of these three orientations, $\{100\}$ silicon wafers are most widely employed for the fabrication of microelectromechanical systems (MEMS) and complementary metal oxide semiconductor (CMOS) devices. In the fabrication of MEMS, alkaline solution (e.g. potassium hydroxide $(\mathrm{KOH})$, tetramethylammonium hydroxide (TMAH), etc.) based silicon anisotropic etching is frequently used to make a wide range of microstructures in silicon wafers [1-10]. In this etching method, as shown in Figure 1, undercutting occurs at mask patterns containing the extruded (or convex) corners [11]. The shape of the corner obtained after undercutting is determined using a polar diagram of lateral underetch rates as illustrated in Figure 1 [12]. The corner undercutting also takes place on $\{110\}$ silicon wafers [13-15]. On one hand, undercutting is advantageously used for the releasing of microstructures (e.g. cantilever beam), but on the other hand, it is undesirable for the realization of mesa structures, bent V-grooves, proof mass for accelerometer, etc. In the TMAH-based anisotropic etchants, undercutting

\footnotetext{
* Correspondence: prem@iith.ac.in

MEMS and Micro/Nano Systems Laboratory, Department of Physics,

Indian Institute of Technology Hyderabad, Hyderabad, India
}

reduces dramatically when a very small amount (e.g. $0.1 \%$ by volume) of surfactant (e.g. Triton-X-100, polyethylene glycol (PEG), NC-200, etc.) is added in the etchant [16-23]. In order to explain the mechanism behind the corner undercutting, several models have been proposed [12,24-27]. They explain that the appearance of high index planes during etching is the main cause of the undercutting. However, these models do not explain very clearly why the undercutting starts at convex corner and why not at concave corners.

In this paper, a simple and robust model is proposed to explain the phenomenon of severe undercutting at the convex corner as well as no-undercutting at the concave corner in wet anisotropic etchants. Moreover, the same model presents why the undercutting is reduced in surfactant added TMAH solution.

\section{Findings}

Figure 2 shows the schematic view of different planes in a unit cell and the shape of mesa structure fabricated on $\mathrm{Si}\{100\}$ wafer surface. The concave and convex corners in a microstructure are illustrated in Figure 3. The $\{111\}$ planes are the most stable (i.e. lowest etch rate) planes in wet anisotropic etchants. However the convex corners (i.e. the intersection of the two $\{111\}$ planes) is still vulnerable to etching and this vulnerability is the reason for extensive undercutting. Now the question is, why the 


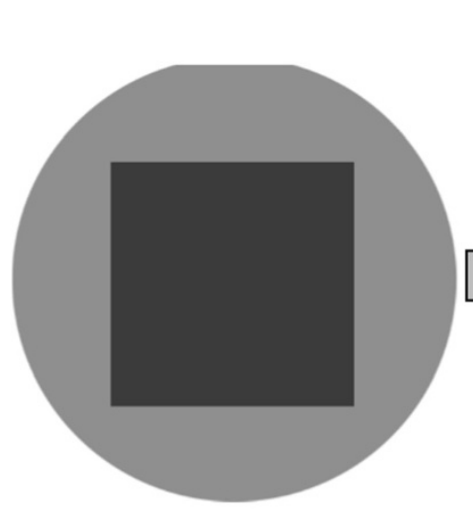

A masked pattern on $\{100\}$ wafer

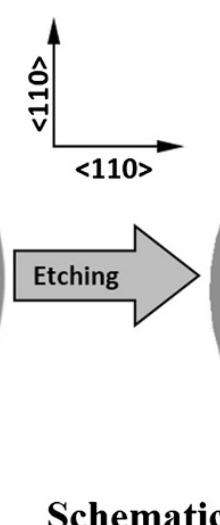

Schematic
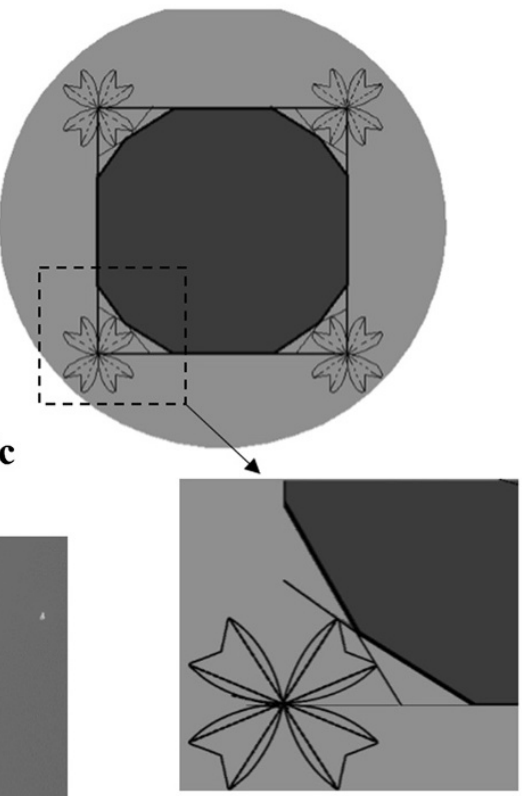

Magnified view of one corner

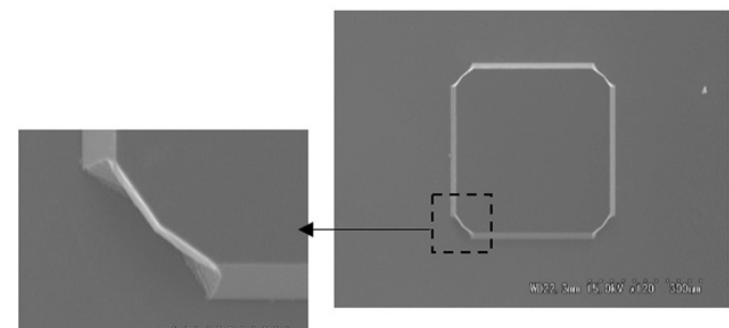

SEM picture

Figure 1 Schematic and SEM pictures of square shaped mask pattern etched in anisotropic etchant on $\{100\}$ Si wafer. Undercutting shape is analyzed using theorientation-dependent lateral underetchrates that are commonly determined by wagon-wheel method [12].

intersecting $\{111\}$ planes at the convex corner are vulnerable? The proposed undercut model answers it on the basis of the fact that the tangent plane on the intersecting $\{111\}$ planes (i.e. the convex corner) is $\{110\}$ as shown in the Figure 2 and this plane exhibits high etch rate in pure $\mathrm{KOH}$ and TMAH solutions $[7,10,11]$. Since the convex corner lies on the $\{110\}$ plane, the etch rate of the corner is much higher than the corresponding $\{111\}$ planes forming the corner and therefore the phenomenon of undercutting is observed at the convex corners. It is to be noted here that the concept of dangling bond is not enough to explain the variation in etching as both the convex corner and the $\{111\}$ plane contain the atoms with only one dangling bond as can be observed in

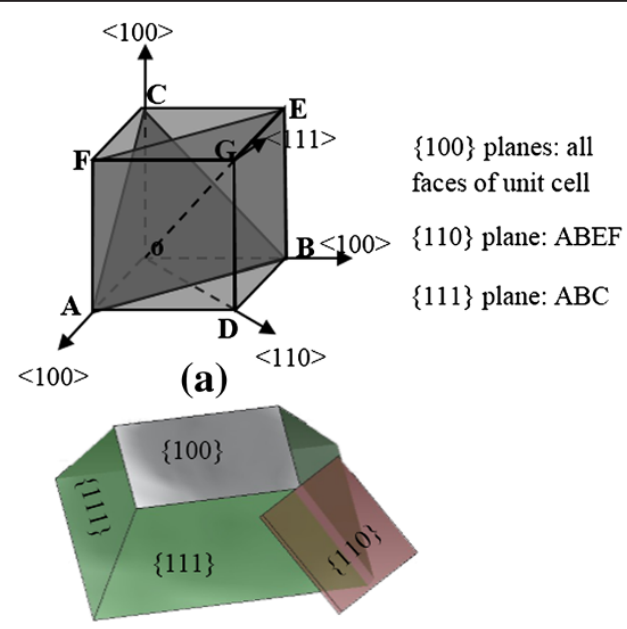

(b)

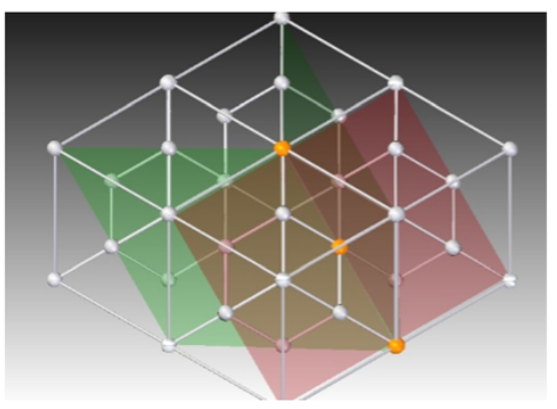

(c)

Figure 2 Schematic view: (a) a unit cell exhibiting three principle planes, (b) $\{110\}$ tangent plane on a convex corner of a mesa structure formed by four convex corners on $\mathrm{Si}\{100\}$ surface, (c) convex corner and its tangent plane in a unit cell. 


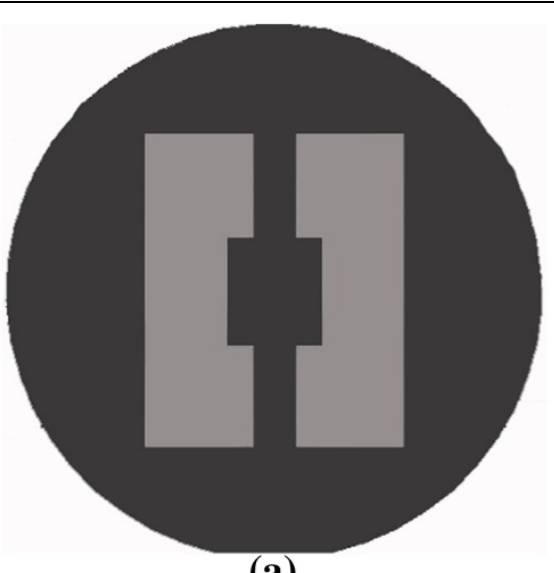

(a)

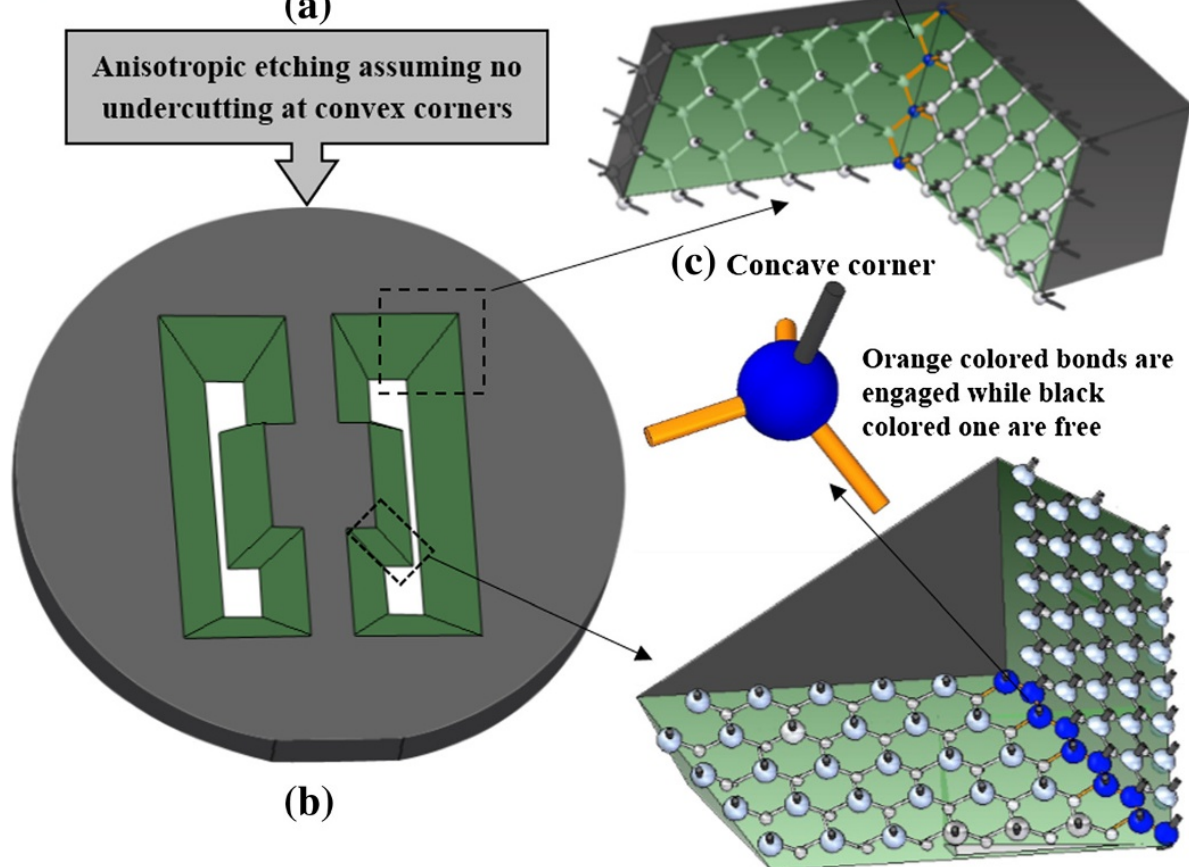

(d) Convex corner

Figure 3 Schematic representation of concave and convex corners: (a) masking pattern on $\{100\}$ Si wafer, (b) anisotropically etched pattern assuming no-undercutting takes place at convex corners, (c) close-up view of concave corner exhibiting silicon atoms of $\{111\}$ plane with one dangling bond, while the atoms belonging to concave corner do not contain any dangling bonds, (d) close-up view of convex corner showing silicon atoms of $\{111\}$ plane and the convex corner with one dangling bond.

Figure 3(d). The etching behavior of tangent plane at convex corner is more appropriate to describe the etching characteristic of convex corner in anisotropic etchants.

One more interesting question which arises here is that why there is no undercutting observed at the concave corner, even though these corners are also formed by the intersection of two $\{111\}$ planes. If we closely observe the concave and convex corners (Figure 3), we can easily notice the difference in the bond structure at the intersecting edge. The silicon atoms belonging to a convex edge consist of dangling bonds (Figure 3(d)), while the atoms belonging to the concave corner (Figure 3(c)) do not contain any dangling bond (i.e. all the bonds are engaged). The reason behind this is that the crystallographic structure of silicon arranges the atoms at the two types of corners (i.e. convex and concave) in such a way that the convex edged silicon atoms have one dangling bond, while the concave cornered silicon atoms have all their bonds engaged and thus there is no dangling bond. The absence of the dangling bond at the concave corner restricts undercutting, however the etching occurs parallel to the $\{111\}$ planes. This is a very simple way of explaining why a convex corner is more vulnerable to undercutting whereas there is no-undercutting at the concave corner.

Now the last concern is, why does the undercutting at convex corner decrease dramatically in surfactant-added 
TMAH [16-23]. The summary of the etching characteristics of the pure and very small amount of surfactant ( $0.1 \%$ by volume) added TMAH solution is presented in Figure 4 . The etch rate of $\{100\} \mathrm{Si}$ is almost the same in pure and surfactant-added TMAH (Figure 4(a), while the etch rate of $\{110\}$ silicon is decreased to a considerably low level when a very small amount of surfactant is added into the etchant (Figure 4(b)). As can be seen in Figures 4(c)-4(e), the phenomenon of undercutting is reduced significantly when etching is carried out in Triton-X-100 (i.e. surfactant) added TMAH.

The surfactant molecules form an adsorbed monolayer film on the surface with the hydrophobic part of the molecules (or head) in contact with the hydrophobic surface while the hydrophilic part of the molecules (or tail) remains in contact with water $[22,28]$. Hence the surfactant molecules adsorb more densely on more hydrophobic surface. The relative hydrophobicity of the

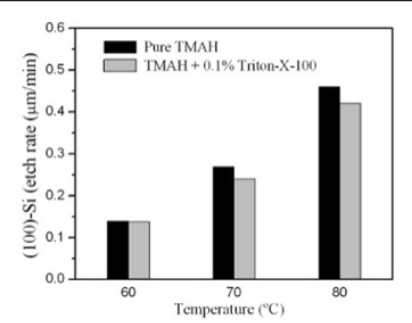

(a)

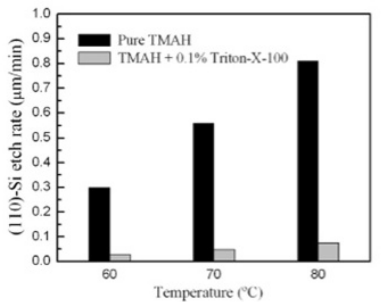

(b)
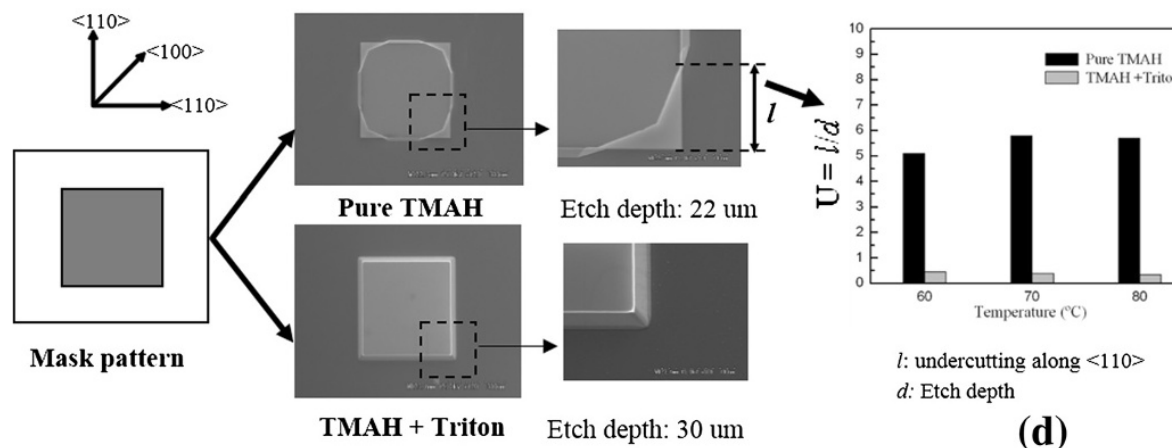

(c) SEM pictures of convex corners

(d)

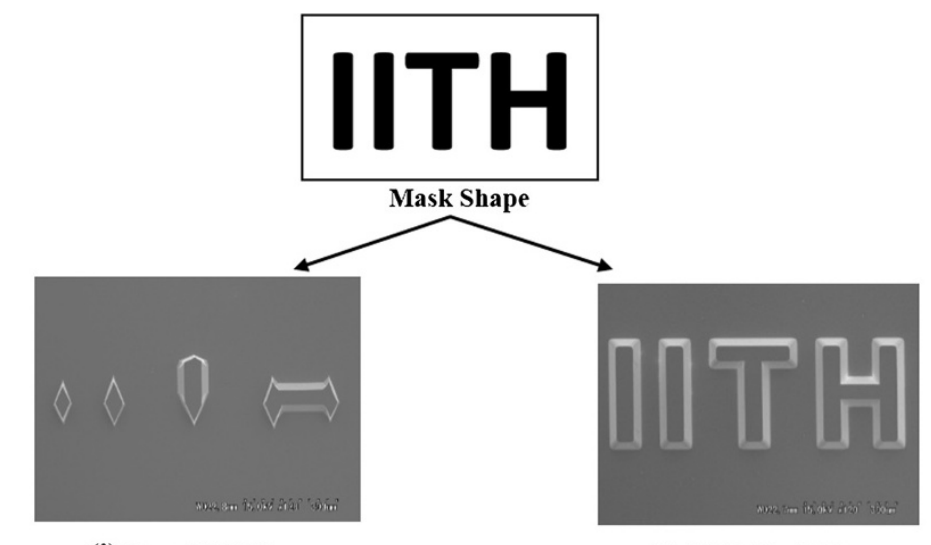

(i) Pure TMAH

Etch depth: 30 um

(ii) TMAH + Triton

(e) SEM pictures of wet bulk micromachined alphabet letters containing concave and convex corners

Figure 4 Etching characteristics of pure and surfactant (Triton X-100) added 25 wt\% TMAH: (a) Si\{100\} etch rate at different temperatures; (b) Si $\{110\}$ etch rate at different temperatures; (c) etched profiles of convex corners; (d) undercutting $(U=I / d)$ at convex corners. (e) Micromachining of alphabets in $\{100\} S i$ surface using (i) pure TMAH and (ii) TMAH+Triton. 
silicon surface can be estimated by the density of $\mathrm{H}$ terminations. The $\mathrm{Si}\{110\}$ is more hydrophobic (lower H-density) than $\mathrm{Si}\{110\}$, resulting in the formation of a more densely packed surfactant layer [22]. Several studies have been performed to confirm the orientation dependent adsorption of surfactant molecules $[18,19,22]$. They explain that the maximum adsorption is for $\{111\}$ surface followed by $\{110\}$ and $\{100\}$ surfaces. It can be observed from Figure $4(\mathrm{a})$ that the etch rate of $\{100\}$ is almost unaffected when the surfactant is incorporated in the etchant. It means that the layer of surfactant molecules on $\{100\}$ surface is not able to protect the surface from the etchant and thus etch rate is almost unaffected. In the case of $\mathrm{Si}\{110\}$ (Figure 4(b)), significant reduction in the etch rate indicates that the adsorbed layer of surfactant molecules partially protects the surface from the direct attack of reactants during etching.

In order to emphasize the effect of undercutting on the resultant shape of the fabricated structure, the SEM pictures of alphabets "IITH", which comprise concave and convex corners, etched in pure and surfactantadded TMAH are shown in Figure 4(e)(i) and 4(e)(ii), respectively. The letters micromachined in pure TMAH have lost their shapes, while they retain their shapes when etching is performed in surfactant added TMAH. This kind of etching behavior can be easily understood through the proposed undercutting model as it is based on the presence of $\{110\}$ tangent plane at the convex corner i.e. the silicon atoms of convex corner belong to $\{110\}$ plane. As shown in Figure 5, the surfactant molecules form a dense layer on the convex corner. This dense layer inhibits the etchant to react chemically with the silicon atoms at the corner that results in dramatic reduction in the undercutting. It means that when the surfactant molecules adsorb very densely, the surface area available for the etchant to react reduces significantly and thus undercutting rate is remarkably suppressed. On the other hand, the $\{100\}$ plane is not able to attract the surfactant molecules more compactly owing to its less hydrophobicity and thus the etchant can easily react chemically with the silicon atoms and leads to almost the same etch rate as shown in Figure 4(a).

\section{Conclusions}

A simple model is proposed to explain the etching characteristics of convex and concave corners on $\operatorname{Si}\{110\}$ surface in wet anisotropic etchants. The proposed model explains the basic reasons behind the corner undercutting in a very simple and robust way. Moreover, it explains the dramatic reduction in the undercutting in surfactant-added TMAH solution. In the case of the surfactant-added TMAH, crystallographic orientation dependent adsorption of the surfactant molecules (owing to varying hydrophobicity of the crystallographic planes)

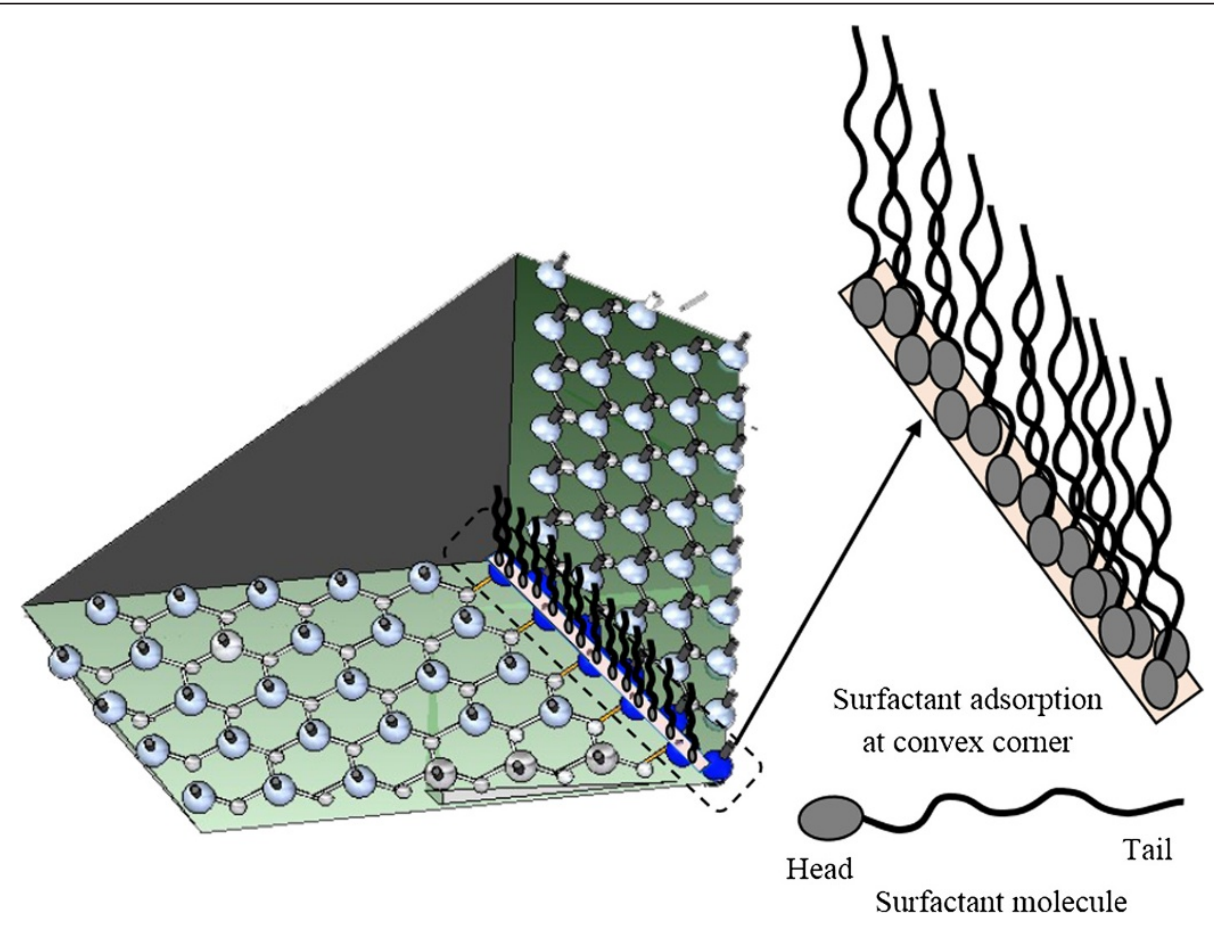

Figure 5 Schematic representation of surfactant adsorption on silicon atoms of convex corners, which belong to $\{110\}$ planes, in surfactant-added TMAH solution during etching process. The dense layer of surfactant molecules protect the convex corners from etchant that result in the reduction of undercutting. 
is employed to describe the significant reduction in the undercutting at convex corners. In order to explain the reason of no-undercutting at concave corners, the role of dangling bonds in etching mechanism is exploited. The undercutting at convex corners is explained using the etch rate behavior of tangent plane appearing at this type of corners.

\section{Competing interests}

The authors declare that they have no competing interests.

\section{Authors' contributions}

PP performed the experiments, analyzed the experimental results and prepared the manuscript. SSS developed 3-D and 2-D models for better visualization of theory and contributed significantly during the preparation of manuscript. All authors read and approved the final manuscript.

\section{Acknowledgments}

This work was supported by research grant from the Department of Science and Technology (Project No. SR/S3/MERC/072/2011), New Delhi, India.

Received: 10 March 2013 Accepted: 27 May 2013

Published: 6 August 2013

\section{References}

1. Kang I, Haskard MR, Samaan ND (1997) A study of two-step silicon anisotropic etching for a polygon-shaped microstructure using $\mathrm{KOH}$ solution. Sens Actuators A62:646-651, http://dx.doi.org/10.1016/50924-4247 (97)01500-8

2. Jiang Y, Liu G, Zhou J (2009) A novel process for circle-like 3D microstructures by two-step wet etching. J Micromech Microeng 19015005:5. doi:10.1088/0960-1317/19/1/015005

3. Kwon JW, Kim ES (2002) Multi-level microfluidic channel routing with protected convex corners. Sens Actuators A97-98:729-733. doi:10.1016/ S0924-4247(02)00012-2

4. Rao MP, Aimi MF, MacDonald NC (2004) Single-mask, three-dimensional microfabrication of high-aspect-ratio structures in bulk silicon using reactive ion etching lag and sacrificial oxidation. Appl Phys Lett 85:6281-6283, http://dx.doi.org/10.1063/1.1834720

5. Lee S, Park S, Cho D (1999) The surface/bulk micromachining (SBM) process: a new method for fabricating released microelectromechanical systems in single crystal silicon. J Microelectromech Syst 8:409-416. doi:10.1109/ 84.809055

6. Chu HY, Fang W (2004) A vertical convex corner compensation and non \{111\} crystal planes protection for wet anisotropic bulk micromachining process. J Micromech Microeng 14:806-813. doi:10.1088/0960-1317/14/6/007

7. Zubel I, Kramkowska M (2005) Possibilities of extension of 3D shapes by bulk micromachining of different Si $(h \mathrm{k} /)$ substrates. J Micromech Microeng 15:485-493. doi:10.1088/0960-1317/15/3/008

8. Yang EH, Yang SS, Han SW, Kim SY (2005) Fabrication and dynamic testing of electrostatic actuators with $\mathrm{p}+$ silicon diaphragms. Sens Actuators A50:151-156. doi:10.1016/0924-4247(96)80100-2

9. Gianchandani YB, Najafi K (1992) A bulk silicon dissolved wafer process for microelectromechanical devices. J Microelectromech Syst 1:77-85. doi:10.1109/84.157361

10. Pal P, Sato K (2009) Complex three dimensional structures in Si\{100\} using wet bulk micromachining. J Micromech Microeng 19:105008 (9pp). doi:10.1088/0960-1317/19/10/105008

11. Pal P, Sato K, Chandra S (2007) Fabrication techniques of convex corners in a (100)-silicon wafer using bulk micromachining: A review. J Micromech Microeng 17:R111-R133. doi:10.1088/0960-1317/17/10/R01

12. Trieu HK, Mokwa W (1998) A generalized model describing corner undercutting by the experimental analysis of TMAH/IPA. J Micromech Microeng 8:80-83. doi:10.1088/0960-1317/8/2/009

13. Jia C, Dong W, Liu C, Zhang X, Zhou J, Zhong Z, Xue H, Zang H, Xu B, Chen W (2006) Convex corners undercutting and rhombus compensation in $\mathrm{KOH}$ with and without IPA solution on (110) silicon. Microelectronics J 37:1297-1301. doi:10.1016/j.mejo.2006.07.008
14. Kim B, Cho DD (1998) Aqueous KOH etching of silicon (110) etch characteristics and compensation methods for convex corners. J Electrochem Soc 145(7):2499-2508. doi:10.1149/1.1838668

15. Dong W, Zhang X, Liu C, Li M, Xu B, Chen W (2004) Mechanism for convex corner undercutting of (110) silicon in $\mathrm{KOH}$. Microelectronics J 35:417-419, http://dx.doi.org/10.1016/j.mejo.2004.01.005

16. Yang CR, Chen PY, Yang CH, Chiou YC, Lee RT (2005) Effects of various ion-typed surfactants on silicon anisotropic etching properties in $\mathrm{KOH}$ and TMAH solutions. Sens Actuators A15:271-281. doi:10.1016/j.sna.2004.09.017

17. Sekimura M (1999) Anisotropic etching of surfactant-added TMAH solution. In: Proc. 12th IEEE Micro-Electro-Mech. Syst. Conf., Orlando, Florida, pp 650-655. doi:10.1109/MEMSYS.1999.746904

18. Gosálvez MA, Tang B, Pal P, Sato K, Kimura Y, Ishibashi K (2009) Orientation and concentration dependent surfactant adsorption on silicon in aqueous alkaline solutions: explaining the changes in the etch rate, roughness and undercutting for MEMS applications. J Micromech Microeng 19:125011 (18pp). http://www.sciencedirect.com/science/article/pii/ S0924424700003174

19. Tang B, Pal P, Gosalvez MA, Shikida M, Sato K, Amakawa H, Itoh S (2009) Ellipsometry study of the adsorbed surfactant thickness on Si\{110\} and Si $\{100\}$ and the effect of pre-adsorbed surfactant layer on etching characteristics in TMAH. Sens Actuators A156:334-341. doi:10.1016/j sna.2009.10.017

20. Resnik D, Vrtacnik D, Aljancic U, Mozek M, Amon S (2005) The role of Triton surfactant in anisotropic etching of $\{110\}$ reflective planes on (100) silicon. J Micromech Microeng 15:1174-1183. doi:10.1088/0960-1317/15/6/007

21. Sarro PM, Brida D, van der Vlist W, Brida S (2000) Effect of surfactant on surface quality of silicon microstructures etched in saturated TMAHW solutions. Sens Actuators A 85:340-345, http://dx.doi.org/10.1016/50924-4247(00)00317-4

22. Pal P, Sato K, Gosalvez MA, Kimura Y, Ishibashi K, Niwano M, Hida H, Tang B, Itoh S (2009) Surfactant adsorption on single crystal silicon surfaces in TMAH solution: orientation-dependent adsorption detected by In-situ Infra-Red spectroscopy. J Microelectromech Syst 18:1345-1356. doi:10.1109/ JMEMS.2009.2031688

23. Pal P, Sato K, Gosalvez MA (2012) Etched profile control in anisotropic etching of silicon by TMAH+Triton. J Micromech Microeng 22(6):065013 (9pp). doi:10.1088/0960-1317/22/6/065013

24. Chahoud M, Wehmann HH, Schlachetzki A (1998) Etching simulation of convex and mixed InP and Si structures. Sens Actuators A69:251-258. http:// www.sciencedirect.com/science/article/pii/S0924424702000171

25. Schroder $\mathrm{H}$, Obermeier $\mathrm{E}(2000)$ A new model for Si $\mathrm{s}_{100\}}$ convex corner undercutting in anisotropic $\mathrm{KOH}$ etching. J Micromech Microeng 10:163-170. doi:10.1088/0960-1317/10/2/311

26. Shikida M, Nanbara K, Koizumi T, Sasaki H, Sato K, Odagaki M, Ando M, Furuta $\mathrm{S}$, Asaumi K (2002) A model explaining mask-corner undercut phenomena in anisotropic silicon etching: a saddle point in the etching-rate diagram. Sens Actuators A 97-98:758-763, http://dx.doi.org/10.1016/S0924-4247(02)00017-1

27. Chang Chien WT, Chang CO, Lo YC, Li ZW, Chou CS (2005) On the Miller-indices determination of $\mathrm{Si}_{\{100\}}$ convex corner undercut planes. J Micromech Microeng 15:833-842. doi:10.1088/0960-1317/15/4/022

28. Tiberg F (1996) Physical characterization of non-ionic surfactant layers adsorbed at hydrophilic and hydrophobic solid surfaces by time-resolved ellipsometry. J Chem Soc Faraday Trans 92:531-538. doi:10.1039/ FT9969200531

doi:10.1186/2213-9621-1-1

Cite this article as: Pal and Singh: A simple and robust model to explain convex corner undercutting in wet bulk micromachining. Micro and Nano Systems Letters 2013 1:1. 